\title{
Stochastic Renormalization Group in Percolation: I. Fluctuations and Crossover
}

\author{
Martin Z. Bazant ${ }^{\mathrm{a}}$

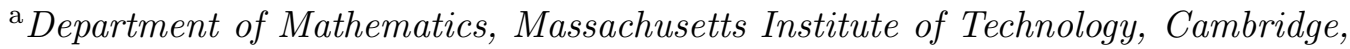 \\ MA 02139
}

\begin{abstract}
A generalization of the Renormalization Group, which describes order-parameter fluctuations in finite systems, is developed in the specific context of percolation. This "Stochastic Renormalization Group" (SRG) expresses statistical self-similarity through a non-stationary branching process. The SRG provides a theoretical basis for analytical or numerical approximations, both at and away from criticality, whenever the correlation length is much larger than the lattice spacing (regardless of the system size). For example, the SRG predicts order-parameter distributions and finite-size scaling functions for the complete crossover between phases. For percolation, the simplest SRG describes structural quantities conditional on spanning, such as the total cluster mass or the minimum chemical distance between two boundaries. In these cases, the Central Limit Theorem (for independent random variables) holds at the stable, off-critical fixed points, while a "Fractal Central Limit Theorem" (describing long-range correlations) holds at the unstable, critical fixed point. This first part of a series of articles explains these basic concepts and a general theory of crossover. Subsequent parts will focus on limit theorems and comparisons of small-cell SRG approximations with simulation results.
\end{abstract}

Key words: Percolation; Spanning; Renormalization group; Branching process; Distribution function; Limit theorem; Finite-size scaling; Crossover.

Dedicated to H. Eugene Stanley on the occasion of his sixtieth birthday

$\overline{1}$ This paper was originally prepared for the proceedings of the conference, "Horizons in Complex Systems," held in Messina, Sicily in December 2001 to commemorate Prof. Stanley's birthday.

Preprint submitted to Elsevier Preprint June 10, 2002 


\section{Introduction}

The theory of critical phenomena is mostly concerned with phase transitions in the thermodynamic limit of infinite systems. In this limit, an order parameter has unambiguous values in each phase and is singular at a precise critical point of the control variable (temperature, concentration, etc.) [1-4]. In finite systems, however, there are two basic modifications to the thermodynamic picture: (i) The critical point becomes blurred since all statistical averages are analytic functions of the control variable, and (ii) the order parameter fluctuates around its mean value. Accordingly, the order parameter in a finite system is described by a probability distribution, which depends non-trivially on the control variable and the system size.

As arguably the simplest critical phenomenon, percolation [5-7] provides a natural setting within which study order-parameter fluctuations in finite systems. Moreover, in recent years there has been growing practical interest in the distribution functions of structural quantities, such as the minimum or maximum chemical distance between two terminals [8-17], the masses of spanning clusters [11-13,18], and the mass of the largest cluster [19-22]. These random quantities not only act as order parameters for the phase transition, but also play a crucial role in both dynamical $[23,24,16]$ and mechanical $[19,21]$ properties of heterogeneous materials [25].

There is no existing theory to predict order-parameter statistics for arbitrary values of concentration and system size, but considerable progress has been made in understanding the limiting behavior in each thermodynamic phase. Classical limit theorems for independent random variables hold in the normal phases, where correlations are negligible (by definition). In the supercritical phase, the masses of large fragments of the infinite cluster [26-28] and other structural quantities $[29,30]$ satisfy the Central Limit Theorem, i.e. the distribution is Gaussian, and the mean and variance both grow linearly with the system size. In the subcritical phase, the mass of the largest cluster is governed by the theory of extremes of independent random variables $[19,20]$. In this case, the distribution has the Fisher-Tippett (or Gumbel) form with non-universal corrections due to discrete-lattice effects, while the mean grows logarithmically with system size (with a bounded variance) [20].

Computer simulations of percolation have shown that various order parameters in the critical phase have different universal distributions with stretchedexponential tails (when scaled to have unit mean) $[8,9,11,18,14-16,22]$. These signatures of what we call the "Fractal Central Limit Theorem" [31] can be predicted by analyzing stationary branching processes [32-34], as was recently

pointed out by Hovi and Aharony [13]. Aside from an early analysis of meanfield percolation on the Cayley tree [10], these authors made the first (and, 
to the author's knowledge, only) systematic use of branching processes to approximate critical limiting distributions, although they focused mainly on the tails of spanning-cluster mass distributions [13].

In this first part of a series of articles, we present the "Stochastic Renormalization Group" (SRG), a theoretical formalism based on non-stationary branching processes which describes the crossover of order-parameter distributions between different thermodynamic phases. The SRG expresses the statistical self-similarity of finite systems in which the correlation length, $\xi$, and linear system size, $L$, are both much larger than the lattice spacing, $a$. The critical $(\xi \gg L)$ and off-critical $(\xi \ll L)$ fixed points of the SRG correspond to various limit theorems for the order parameter. In the latter case, small correlation lengths $(\xi \approx a)$ are also allowed. Although the basic idea of the SRG applies to critical phenomena and random fractals in general, we develop it here on the specific context of percolation.

The article is organized as follows. In section 2, we set the stage by reviewing classical renormalization-group ( $\mathrm{RG}$ ) concepts in percolation. In section 3, we present the SRG for any statistical quantity conditional on spanning the system, such as the total mass of spanning clusters or the minimum chemical distance. In section 4, we show how the SRG generally describes crossover. In sections 5 and 7 , we briefly explain why these the off-critical and critical fixed points are associated with the CLT and the FCLT, respectively. In section 8, we summarize the SRG "flow" in the space probability distributions for percolation quantities dependent upon spanning, and we conclude by discussing the general relevance of the SRG for fractals and phase transitions in finite systems. Subsequent parts will analyze the fixed points in detail, including more comparisons with simulation data. The subtle case of the largest cluster (which may not span), the most natural order parameter in finite systems [19,20,35-37], will also be addressed.

\section{RG Concepts in Percolation}

\subsection{Self-Similarity at the Critical Point}

The basic idea behind all RG methods for critical phenomena is the statistical self-similarity of large systems near a critical point [2-4]. This essentially follows from dimensional analysis [38]: In the limit $a / L \rightarrow 0$ and $a / \xi \rightarrow 0$, there are only two (out of originally three) relevant length scales, $L$ and $\xi$, so all systems with the same dimensionless ratio, $L / \xi$, must have approximately the same statistical properties (upon a suitable normalization). The self-similarity becomes exact in the thermodynamic limit, $L \rightarrow \infty$, as the critical point is 
approached, $\xi \rightarrow \infty$, with the scaling variable, $L / \xi$, held constant.

The notion of self-similarity is exploited by the RG to calculate nontrivial properties of the critical point, which cannot be obtained by simple dimensional analysis. For example, in the case of percolation, according to the arguments above, a large system of $N=m n$ sites must have approximately the same connectivity statistics as a coarse-grained system of $n \gg 1$ large "cells" (or "blocks") containing $m \gg 1$ sites each, provided that an effective occupation probability for cells, $p_{m}=R_{m}(p)$, is defined so as to preserve the dimensionless ratio, $L / \xi$ :

$$
\frac{(m n)^{1 / d}}{\xi(p)}=\frac{n^{1 / d}}{\xi\left(p_{m}\right)} .
$$

where $d$ is the system dimension $\left(N=L^{d}\right)$. This is analogous to Kadanoff's original block renormalization of the coupling constant for magnetic spins [4].

If the exact dependence of the correlation length on $p$ were known, then the cell occupation probability would be given by

$$
R_{m}(p)=\xi^{-1}\left(\frac{\xi(p)}{m^{1 / d}}\right)
$$

The trick is to now reverse the logic: From a suitable definition of the cell occupation probability, an estimate of the correlation length, $\xi^{(n)}(p)$, can be obtained by successively "renormalizing" the occupation probability,

$$
p_{m n}=R_{n}\left(p_{m}\right)
$$

starting from $p_{1}=p$. By construction, an appropriate choice of $R_{n}(p)$ yields a valid asymptotic approximation in the large-cell $\operatorname{limit} \lim _{n \rightarrow \infty} \xi^{(n)}(p)=\xi(p)$, although it is not trivial to make this choice for a given problem such that the convergence is fast enough to allow the use of small cells.

\subsection{Asymptotic Approximations}

Estimates of correlation length and $p_{c}$ itself are obtained from the RG, Eq. (3), as follows. As shown in Fig. 1, for any suitable choice of $R_{n}(p)$, the recursion (3) converges to one of three possible fixed points in the thermodynamic limit, 


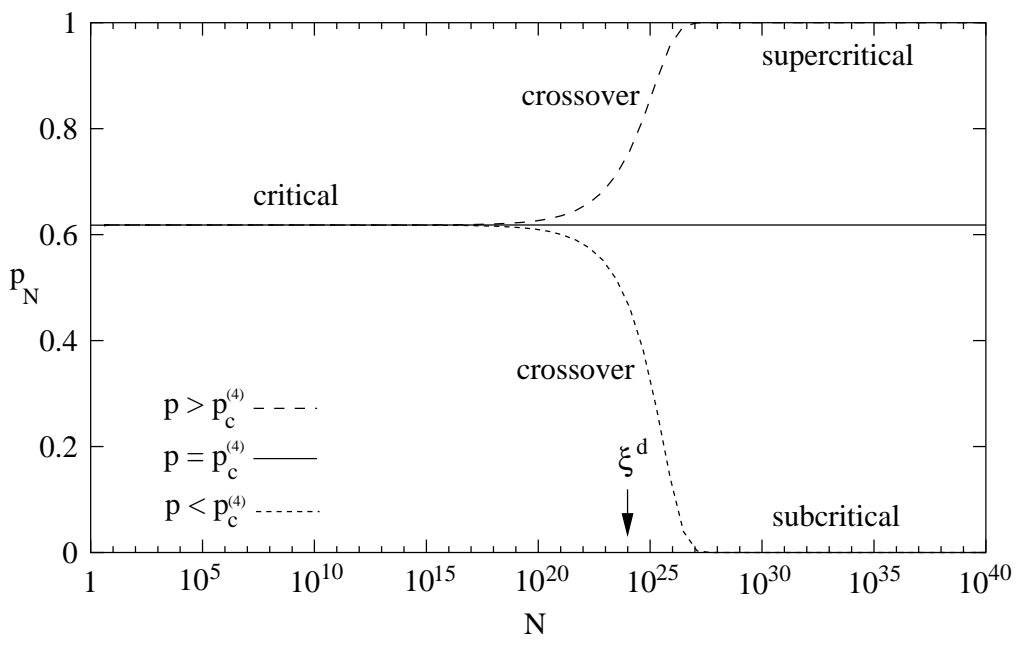

Fig. 1. Typical trajectories of the renormalized cell occupation probability $p_{N}$ given by Eq. (3) for the four-site $\left(N=4^{j}\right)$ RG scheme described below in Eq. (11) starting at the critical fixed point, $p_{1}=p_{c}^{(4)}=(\sqrt{5}-1) / 2$ (solid line), a nearby subcritical value, $p_{1}^{\prime}=p_{c}^{(4)}-10^{-8}$ (dotted line), and a nearby supercritical value, $p_{1}^{\prime \prime}=p_{c}^{(4)}+10^{-8}$ (dashed line). An associated estimate of the correlation length, $\xi^{(4)}\left(p_{1}^{\prime}\right)$, in the subcritical case is labeled as the system size $N=\xi^{d}$ where the renormalized occupation probability $p_{N}$ drops below 0.5 . This is very close to a correlation length estimate, $\xi^{(4)}\left(p_{1}^{\prime \prime}\right)$, for the supercritical case where $p_{N}$ rises above 0.7 .

$j \rightarrow \infty$ :

$$
\lim _{j \rightarrow \infty} p_{n^{j}}= \begin{cases}0 & \text { for } 0 \leq p<p_{c}^{(n)} \\ p_{c}^{(n)} & \text { for } p=p_{c}^{(n)} \\ 1 & \text { for } p_{c}^{(n)}<p \leq 1\end{cases}
$$

the stable subcritical fixed point, $p=0$; the stable supercritical fixed point, $p=1$; or the unstable critical fixed point, $p=p_{c}^{(n)}$, which is an $n$-cell estimate of the true infinite-system value of $p_{c}$. As long as the RG transformation, is chosen to satisfy

$$
\lim _{n \rightarrow \infty} R_{n}(p)=\left\{\begin{array}{l}
0 \text { for } 0 \leq p<p_{c} \\
c \text { for } p=p_{c} \\
1 \text { for } p_{c}<p \leq 1
\end{array}\right.
$$

for some constant $0 \leq c \leq 1$, then the $\mathrm{RG}$ approximation converges in the large-cell limit, $\lim _{n \rightarrow \infty} p_{c}^{(n)}=p_{c}$. As shown in Fig. 1 , for $p \neq p_{c}^{(n)}$, an estimate of the correlation length, $\xi^{(n)}(p)$, can be defined as the (interpolated) linear system size, $n^{j / d}$, where the cell occupation probability, $p_{n^{j}}$, first passes within a given tolerance of a stable fixed point as $j \rightarrow \infty$. 
The RG introduces a useful artificial length scale, the linear cell size, $b\left(n=b^{d}\right)$. In order for the lattice-spacing $a$ to not affect the RG calculation of critical behavior, we must have $b \gg a$, but the underlying assumption of self-similarity also requires $b \ll \xi(p)$, which is guaranteed near the critical point, where $\xi\left(p_{c}\right)=\infty$. General considerations of dimensional analysis imply that such a divergence must have the form of a power law, $\xi(p) \sim\left(p-p_{c}\right)^{-\nu}$ (e.g. for $p \rightarrow p_{c}^{+}$), but dimensional analysis alone cannot determine the critical exponent $\nu$. The RG, however, easily produces an asymptotic estimate, $\nu^{(n)}$, for a given choice of $n=b^{d}$.

Substituting an approximation of the correlation-length singularity,

$$
\xi^{(n)}(p) \sim\left(p-p_{c}^{(n)}\right)^{-\nu^{(n)}}
$$

into Eq. (1) with $m=n$ yields

$$
R_{n}(p)-p_{c}^{(n)} \sim n^{1 / d \nu^{(n)}}\left(p-p_{c}^{(n)}\right)
$$

from which we identify, $n^{1 / d \nu^{(n)}}=R_{n}^{\prime}\left(p_{c}^{(n)}\right)$. The resulting RG estimate of the critical exponent is

$$
\nu^{(n)}=\frac{1}{\log _{b} R_{n}^{\prime}\left(p_{c}^{(n)}\right)} .
$$

(Note that, since $R_{n}(p)$ is generally a polynomial, it is analytic at $p_{c}^{(n)}$, which implies that the correlation length diverges with the same exponent for $p \rightarrow$ $p_{c}^{+}$and $p \rightarrow p_{c}^{-}$.) Such RG estimates of critical exponents are believed to converge to their exact values in the large-cell limit, provided that Eq. (5) holds, consistent with our analysis below and extensive numerical evidence in the literature.

\subsection{The Renormalization Group}

Algebraically, the recursion in Eq. (3) represents a group of operators acting on the occupation probability (more precisely, a semigroup without an inverse.) There are at least two equivalent ways to construct this "renormalization group". First, by using the same number of cells at each level of recursion, the renormalized occupation probabilities, $p_{n^{j}}=\mathcal{R}_{j}^{(n)} p$, for the sequence of system sizes, $N=n, n^{2}, n^{3}, n^{4}, \ldots$, can be generated by iterates of the function, $R_{n}(p)$, acting on the site occupation probability, $p \in[0,1]$. In this case, the group 
operators,

$$
\mathcal{R}_{j}^{(n)}=R_{n}^{\circ j}=R_{n} \circ R_{n} \circ \ldots \circ R_{n}
$$

(j compositions of $R_{n}(p)$ ) are mappings from $[0,1]$ to $[0,1]$ which depend on the cell size, $n$.

Alternatively, one could view $R_{n}(p)$ as the initial condition for a group of operators acting on the space of functions, $\{f:[0,1] \mapsto[0,1]\}$, by setting the number of cells to equal the number of sites at each iteration. For example, the renormalized probabilities, $p_{n^{2 j}}=\mathcal{R}_{j} R_{n}(p)$, for the sequence of system sizes, $N=n, n^{2}, n^{4}, n^{8}, \ldots$, can be generated by group operators defined as follows,

$$
\begin{aligned}
\mathcal{R}_{j+1} & =\mathcal{R}_{j} \mathcal{R}_{1} \\
\mathcal{R}_{1} f & =f \circ f .
\end{aligned}
$$

Although the initial condition depends on the function $R_{n}(p)$, in this case the $\mathrm{RG}$ is always the same. As such, broad sets of initial conditions can yield the same limiting behavior, and in this way the RG explains, at least qualitatively, the universality of critical exponents.

\subsection{Cell Occupation by Spanning}

These abstract concepts would not be terribly important were it not for the fact that they can be put to practical use by clever definitions of the cell occupation probability. In the spirit of this commemorative issue, we focus on the seminal contributions of Reynolds, Stanley, and Klein [39-41]. Before their work, a few simple, small-cell decimation procedures were proposed [4245], but these authors first defined a cell to be "occupied" it is spanned by a cluster from one side to another in at least one direction. This choice captures the essence of percolation as a phase transition in connectivity. Moreover, it should always produce convergent RG approximations because Eq. (5) holds rigorously for any spanning rule that, as $n \rightarrow \infty$, requires a cluster to extend across an infinite distance [6].

For a given choice of spanning rule, the spanning probability, $R_{n}(p)$, can be evaluated exactly for small cells or approximately by Monte-Carlo simulation for large cells [39-41]. For example, for a square site or bond lattice in two dimensions, the function $R_{n}(p)$ could be the probability that a cluster connects opposite boundaries of a square cell in one direction, both directions, or either direction. Once $R_{n}(p)$ is obtained, estimates of $p_{c}$ and $\nu$ are given by the 
expressions above. The fractal dimension $D_{f}$ of incipient infinite clusters can also be estimated by generalizing the RG to include "ghost bonds" between non-nearest neighbor sites, although below we derive a different estimate which does not require any ghost bonds.

For illustration purposes, throughout this paper we will consider the following, very simple example. For site percolation on the square lattice, a reasonable RG for a small cell of only $n=2 \times 2$ sites is based on the probability of spanning in one direction $[40,41]$,

$$
R_{4}(p)=p^{4}+4 p^{3} q+2 p^{2} q^{2}
$$

(where $q=1-p$ ) which is the sum of probabilities to have spanning clusters of size four, three, or two, respectively. Solving the quartic equation, $R_{4}(p)=p$, yields (curiously) the golden mean as an estimate of the critical point,

$$
p_{c}^{(4)}=\frac{\sqrt{5}-1}{2} \approx 0.618034,
$$

which is fairly close to the best available numerical value [46], $p_{c}=0.59274621(13)$. The critical exponent estimate from Eq. (8),

$$
\nu^{(4)}=\frac{1}{\log _{2}\left[4 p_{c}^{(4)}\left(1-\left(p_{c}^{(4)}\right)^{2}\right)\right]} \approx 1.63529,
$$

is not as close to the presumably exact value, $\nu=4 / 3$, although larger cells slowly improve this estimate [41]. Of course, more sophisticated and accurate RG schemes exist for various percolation problems, but this simple example illustrates the power of RG methods to approximate nontrivial quantities, such as the critical exponents and $p_{c}$ itself, with remarkable ease. Using the same example, we will produce RG estimates below for many other quantities, both at and away from the critical point, with little additional effort.

In the limit of large cells, $n \rightarrow \infty$, numerical evidence suggests that RG estimates converge to their exact values (whenever known), and the accuracy of small-cell approximations is often quite remarkable $[39,41,47,13,59]$. In spite of these successes, however, the validity of the large-cell RG based on spanning has recently been debated in the context of critical spanning probabilities [48$56,47,57-59]$. Strictly speaking, Equation (3) seems to predict that the critical spanning probability in the infinite-system limit is precisely equal to $p_{c}$. On the other hand, simulations on the square site lattice support another theoretical prediction (from conformal field theory [60]) that the critical spanning probability in two dimensions is exactly $1 / 2$, which is somewhat less than $p_{c}$. 
There is no contradiction, however, since the RG only predicts that the spanning probability at the $n$-cell estimate $p_{c}^{(n)}$ is equal to $p_{c}^{(n)}$, which need not hold in the limit of infinite cells [47]. In fact, when the effect of the finite

cell size is properly considered as $p_{c}^{(n)} \rightarrow p_{c}$, the RG actually predicts the universal spanning probability, including its subtle dependence on the system shape $[48,60,55,57,58]$ and boundary conditions $[53,54,58]$ with good precision $[47,59]$. In summary, the general validity of the RG based on spanning is now fairly well established, and thus we use it as a starting point for our theory below.

\subsection{Self-Similarity Away From the Critical Point}

The RG is often invoked to motivate (but not to calculate) the "data collapse" of any quantity, $Q(L, p)$ depending on system size $L$ and concentration $p$ onto finite-size scaling functions of the form,

$$
Q(L, p) \sim L^{D_{f}} \Phi\left(\frac{L}{\xi(p)}\right)
$$

where the exponent, $D_{f}$, (which is the fractal dimension, in the case of spanning clusters) and the dimensionless scaling function, $\Phi(x)$, do not depend on $L$ or $p$. (Here and below, we always choose units to set $a=1$.) As explained above, this result is simply a consequence of dimensional analysis.

There is no existing theory to predict finite-size scaling functions. Although the fractal dimension can be calculated from the RG, the scaling function is usually obtained by the ad hoc fitting of numerical data $[5,7,61,20]$. This approach, however, is quite tedious and fails to take advantage of the full power of the RG.

Although RG methods calculations have mostly been applied to the critical phase, where $\xi \gg L$ (or $\xi=\infty$ ), the general arguments given above only require that the correlation length be large, $\xi \gg 1$, but not necessarily larger than the linear system size $L$. Therefore, in principle the $R G$ can not only motivate, but also predict, finite-size scaling functions, as we demonstrate by a suitable generalization below.

For RG approximations using cells of size $n=b^{d}$, the scaling law, Eq. (14), is replaced by

$$
Q(L, p) \sim L_{f}^{D_{f}^{(n)}} \Phi^{(n)}\left(\frac{L}{\xi^{(n)}(p)}\right)
$$


where $\xi^{(n)}(p), \Phi^{(n)}(x), D_{f}^{(n)}$ are estimates based on the $n$-cell level of coarse graining. The scaling function, $\Phi^{(n)}(x)$, can be determined at any value of its argument, $L / \xi^{(n)}(p)$, by an appropriate renormalization of $p$ to maintain this ratio in the limit $N=L^{d}=n^{j} \rightarrow \infty$, as explained below. Since $b / \xi(p) \rightarrow 0$ in this limit, the general arguments above imply convergence, $\lim _{n \rightarrow \infty} \Phi^{(n)}(x)=$ $\Phi(x)$.

If the system size is large, $L \gg 1$, and one is not interested in accurately describing crossover (which also requires being close to the critical point, $\xi \gg 1$ ), then the RG can actually produce accurate approximations far from the critical point, even when the correlation length is small, $\xi(p) \approx 1$. As emphasized in Ref. [20], if a system is partitioned into cells with $b \gg \xi$, then each cell makes independent contributions to any quantity of interest for the whole system, which are easily combined using standard techniques from probability theory. The possibility of accurate approximations in this limit is a consequence of self-similarity near the stable, off-critical fixed points of the RG, where $\xi \ll b \ll L$.

\section{Stochastic Renormalization Group for Spanning-Cluster Masses}

\subsection{The Mathematical Statement of Statistical Self-Similarity}

A key ingredient missing in classical RG methods is the randomness of the order parameter in finite systems. We now reformulate the RG in probabilistic terms for the case of percolation, using a general formalism which could also be applied to other critical phenomena. Let $X_{N}(p)$ be an order parameter conditional on spanning, such as the total mass of spanning clusters or the minimum chemical distance between opposite boundaries, for a large system of size $N \gg 1$ at a given concentration $p$, sufficiently close to the critical point that discrete lattice effects are small, $\xi(p) \gg 1$. Consider coarse-graining such a system of size $N$ into $n$ cells of size $m$.

The powerful constraint of statistical self-similarity discussed above implies that, upon a suitable renormalization of the occupation probability, the random number of occupied cells, $X_{n}\left(p_{m}\right)$, has the same distribution as in a system of size $n$ with the original value of $p$. Unlike the sites or bonds in the original system, however, the coarse-grained cells do not have equal masses. Instead, each occupied cell contributes a random mass, $X_{m}(p)$, sampled independently from the appropriate distribution for a smaller system of size $m$.

This motivates a recursive construction we refer to as the "Stochastic Renormalization Group" (SRG): The total spanning-cluster mass is given by a re- 
cursive random sum of random variables,

$$
X_{m n}(p)=\sum_{i=1}^{X_{n}\left(p_{m}\right)} X_{m}^{(i)}(p)
$$

where $X_{m}^{(i)}$ denotes the $i$ th independent sample from the mass distribution for each cell. Random cluster masses, $X_{n}(p), X_{n^{2}}(p), X_{n^{3}}(p), \ldots$, at the $n$-cell level of approximation can be generated recursively using Eq. (16), which is fully determined by the cell probability distribution,

$$
f_{n}(p, x)=\operatorname{Prob}\left(X_{n}(p)=x\right)
$$

and the cell occupation probability, $R_{n}(p)$, from Eq. (3). These simple quantities can be either calculated exactly for small cells or approximated numerically for large cells. The SRG then extrapolates the distribution to larger finite systems in an arbitrary stage of crossover between phases.

The SRG approximates the statistics of cluster masses through random geometrical constructions, as illustrated in Figure 2. The coarse-graining procedure described above involves discarding contributions from unoccupied cells, and the figure only shows the sites and cells which survive the entire process (from right to left). Equivalently, clusters are created by recursively refining occupied cells (from left to right). In each row, the picture on the right shows a random cluster of sites, and the pictures to the left show the hierarchical structure of connectivity among the sites at different scales of coarse-graining. At scales smaller than the correlation length, these artificially generated clusters are random fractals characteristic of the critical phase, while at larger scales in normal phases they are objects of integer dimension, as explained below. Although the connectivity of these artificial clusters is not required explicitly (as shown in the figure), their mass distributions must nevertheless approach those of the real spanning clusters in each phase in the large-cell limit, purely as a consequence of statistical self-similarity. The crossover between phases can also be described if the correlation length is much larger than lattice spacing.

\subsection{Analogy with Population Growth}

In mathematical terms, Equation (16) describes a stochastic branching process [32-34], which is notably non-stationary since the generating distribution, $f_{n}\left(p_{m}, x\right)$, is different at every stage of recursion. Since the nineteenth century, stationary branching processes have been analyzed as models of population growth, e.g. in the contexts of human family trees [62], animal pop- 

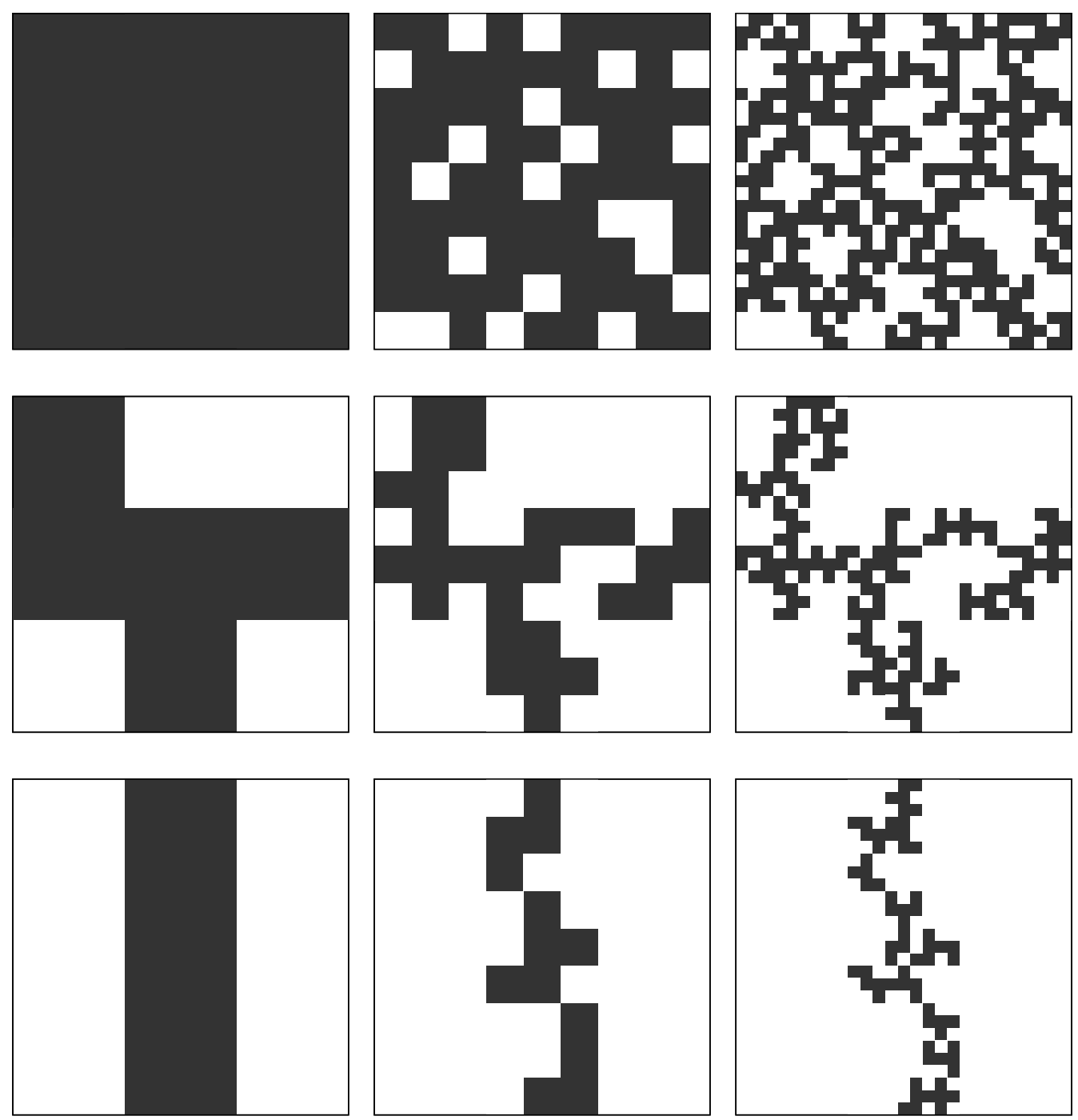

Fig. 2. Three random realizations (in each row from left to right) of a nine-cell SRG branching process for the total cluster mass conditional on spanning in the vertical direction. Top row: the supercritical phase, showing the crossover to critical behavior at scales smaller than the correlation length. Middle row: the critical phase, showing complete statistical self-similarity. Bottom row: the subcritical phase, also showing the crossover to critical behavior. (Note that in these examples the fractal dimension has been reduced well below its actual value for $2 d$ percolation, $D_{f}=91 / 48$, to illustrate the crossover regime with greater clarity.)

ulations [63] and nuclear chain reactions [64]. It does not appear, however, that any mathematical studies have addressed the particular type of nonstationarity in Eq. (16), which has multiple fixed points corresponding to different thermodynamic phases.

The analogy with population growth provides a delightfully simple way to understand the SRG: The hierarchical structure of power-law correlations is analogous to the genealogical structure of populations. This connection is easily understood from the graphical constructions of Fig. 2. Each cluster of sites 
is a graphical representation of a "family tree". The "ancestors" of a site are the coarse-grained cells in which it is contained. From the pictures, it is clear that any random changes to the occupied cells ("ancestors") at a given length scale (or "generation") greatly affect the number of occupied cells and sites ("descendants") at all smaller length scales. Therefore, even though all of the random variables in the branching process are independent, long-range spatial correlations, analogous to "family ties", are induced on the final "population" of sites.

Although the analogy with population growth is conceptually very useful, the SRG branching process involves several new features not typically studied in the context of populations, and thus it requires new mathematical results. For example, the large-cell limit (of infinitely many "children" per individual) is of primary interest for the SRG. More importantly, the structure of spatial correlations would corresponds to a very unusual "family tree" where randomness in the number of children only appears gradually after a certain generation. In the SRG, this occurs when the cell size is comparable to the correlation length (the middle column in Fig. 2). Below this scale, spatial correlations induced on the sites decay as a power law, reflecting the absence of a characteristic length, while above this scale correlations decay exponentially because the number of "children" is no longer random. This gradual variation in the probability distribution for "births" in the branching process causes a phase transition as the linear system size passes through the correlation length, as explained below.

\subsection{Recursions for Distributions and Generating Functions}

Since all the random variables in the SRG branching process are independent, Equation (16) takes the form [32],

$$
f_{m n}(p, x)=\sum_{j=x_{n}^{-}}^{x_{n}^{+}} f_{n}\left(p_{m}, j\right) f_{m}^{* j}(p, x)
$$

in terms of probability distribution functions, where $f^{* j}=f * f * \ldots * f(j$ times) denotes the $j$ th auto-convolution, defined by $f * g(x)=\sum_{y} f(x-y) g(y)$. In general, the bounds of summation, $x_{n}^{-}$and $x_{n}^{+}$, are set by the distribution function, $f_{n}(p, x)$, which also initializes the recursion in Eq. (18). In the case of percolation quantities subject to a spanning condition, the minimum number of occupied cells is of the order of the linear cell size, $x_{n}^{-}=O(b)$, e.g. $x_{n}^{-}=b=$ $n^{1 / d}$ for hypercubic cells, and the maximum number occupied cells is always equal to the total number of cells, $x_{n}^{+}=n$. 
For both analytical and numerical calculations with the SRG, it is convenient to work with various generating functions [32], which are essentially discrete Fourier or Laplace transforms. For example, Equation (18) takes the simple form,

$$
\bar{f}_{m n}(p, z)=\bar{f}_{n}\left(p_{m}, \bar{f}_{m}(p, z)\right)
$$

in terms of probability generating functions,

$$
\bar{f}(z)=\sum_{x=0}^{\infty} f(x) z^{x} .
$$

Other useful forms of the SRG,

$$
\begin{aligned}
\tilde{f}_{m n}(s) & =\tilde{f}_{m}\left(p_{m}, \log \tilde{f}_{m}(p, s)\right) \\
\hat{f}_{m n}(p, s) & =\hat{f}_{n}\left(p_{m}, \hat{f}_{m}(p, s)\right)
\end{aligned}
$$

are expressed in terms of moment generating functions,

$$
\tilde{f}(s)=\bar{f}\left(e^{s}\right),
$$

or cumulant generating functions,

$$
\hat{f}(s)=\log \tilde{f}(s),
$$

respectively. These transformations reduce the complicated sum of convolutions in Eq. (18) to a simple composition of generating functions. In physics parlance, this amounts to changing the representation of the SRG from real space to various definitions of reciprocal (or momentum) space.

\subsection{The Stochastic Renormalization Group}

Let us consider the algebraic structure of the transformations involved in the branching process, Eq. (16). There are various ways to iterate the recursions above, as we now illustrate using the cumulant-generating-function representation, Eq. (22). For example, as in Eq. (9) the same number of cells $n$ can be used at each level of recursion, which yields approximations,

$$
\hat{f}_{n^{j}}(p)=\hat{f}_{n}\left(p_{n^{j-1}}\right) \circ \hat{f}_{n}\left(p_{n^{j-2}}\right) \circ \ldots \circ \hat{f}_{n}\left(p_{n}\right) \circ \hat{f}_{n}(p)
$$


for the sequence of system sizes, $N=n, n^{2}, n^{3}, n^{4}, \ldots$ Alternatively, as in Eq. (10) the number of cells can be set equal to the number of sites at each level of recursion, which yields the (identical) approximations

$$
\begin{aligned}
\hat{f}_{n^{2}}(p) & =\hat{f}_{n}\left(p_{n}\right) \circ \hat{f}_{n}(p) \\
\hat{f}_{n^{4}}(p) & =\hat{f}_{n^{2}}\left(p_{n^{2}}\right) \circ \hat{f}_{n^{2}}(p) \\
& =\hat{f}_{n}\left(p_{n^{3}}\right) \circ \hat{f}_{n}\left(p_{n^{2}}\right) \circ \hat{f}_{n}\left(p_{n}\right) \circ \hat{f}_{n}(p) \\
& \vdots \\
\hat{f}_{n^{2 j+1}}(p) & =\hat{f}_{n^{2}}\left(p_{n^{2}}\right) \circ \hat{f}_{n^{2^{j}}}(p) \\
& =\hat{f}_{n}\left(p_{n^{2^{j+1}}-1}\right) \circ \hat{f}_{n}\left(p_{n^{2^{j+1}}-2}\right) \circ \ldots \circ \hat{f}_{n}\left(p_{n}\right) \circ \hat{f}_{n}(p)
\end{aligned}
$$

for the sequence of system sizes, $N=n, n^{2}, n^{4}, n^{8}, \ldots$ At a fixed point, the same set of RG operators $\left\{\mathcal{R}_{j}\right\}$ as in Eq. (10) now acts on initial conditions, $\hat{f}_{n}(p, s)$, in the space of functions, $\{f:[0,1] \times \mathcal{C} \mapsto \mathcal{C}\}$.

If $p$ is not a fixed point, then the functional transformations are somewhat more complicated, but they still have the structure of an Abelian semigroup (hence the name "Stochastic Renormalization Group") in terms of a set of operators, $\left\{\mathcal{R}_{j}^{S R G}\right\}$, defined by

$$
\begin{aligned}
\mathcal{R}_{j+1} & =\mathcal{R}_{1}^{S R G} \mathcal{R}_{j}^{S R G}, \\
\mathcal{R}_{1}^{S R G} \cdot\{R, f(p)\} & =\{R \circ R, f(R(p)) \circ f(p)\} .
\end{aligned}
$$

These functional operators act on initial conditions, $\left\{R_{n}(p), \hat{f}_{n}(p, s)\right\}$, in the function space, $\{R:[0,1] \mapsto[0,1]\} \times\{\hat{f}:[0,1] \times \mathcal{C} \mapsto \mathcal{C}\}$,

$$
\left\{R_{n^{2^{j}}}(p), \hat{f}_{n^{2^{j}}}(p, s)\right\}=\mathcal{R}_{j}^{S R G}\left\{R_{n}(p), \hat{f}_{n}(p, s)\right\},
$$

where $R_{n}(p)$ is the spanning probability and $\hat{f}_{n}(p, s)$ is the cumulant generating function for the spanning cluster mass in a cell of size $n$.

The fact that the SRG can be expressed as a universal group acting on a space of initial conditions suggests that the crossover of distributions between different phases shares the same universality properties as the fixed points. Therefore, the shapes of scaling functions and the limiting distributions in the critical and crossover regimes should depend on embedding dimension, but not on the microscopic details of the lattice (although we do not claim to rigorously prove this universality here, even within the SRG formalism). Below, we will argue that boundary conditions also affect the universal limiting distributions and thus also the scaling functions for crossover. 


\section{A Theory of Crossover Phenomena}

\subsection{Two Types of Fluctuations}

It is straightforward to derive recursions for the moments,

$$
\mu_{n, k}(p)=\left\langle X_{n}(p)^{k}\right\rangle=\left(z \frac{\partial}{\partial z}\right)^{k} \bar{f}_{n}(p, 1)=\left(\frac{\partial}{\partial s}\right)^{k} \tilde{f}_{n}(p, 0)
$$

or the cumulants,

$$
c_{n, k}(p)=\left(\frac{\partial}{\partial s}\right)^{k} \hat{f}_{n}(p, 0)
$$

by differentiating the various generating-function recursions above. For example, the mean, $\mu_{n}(p)=\mu_{n, 1}(p)=c_{n, 1}(p)$, satisfies the recursion,

$$
\mu_{m n}(p)=\mu_{n}\left(p_{m}\right) \mu_{m}(p)
$$

which simply confirms our intuition that the mean total mass is equal to the mean number of occupied cells times the mean mass per cell. The variance, $\sigma_{n}(p)^{2}=c_{n, 2}(p)$, satisfies another recursion,

$$
\sigma_{m n}(p)^{2}=\mu_{n}\left(p_{m}\right) \sigma_{m}(p)^{2}+\sigma_{n}\left(p_{m}\right)^{2} \mu_{m}(p)^{2},
$$

which is coupled to that of the mean.

The recursion for the variance, Eq. (31), has an important physical interpretation. The first term on the right side is the variance within each cell times the mean number of occupied cells. This represents the well-known additivity of variance for sums of a fixed number of independent random variables. The second term is the additional variance due to fluctuations in the number of occupied cells. As explained above, this kind of fluctuation induces hierarchical (power-law) correlations between the microscopic site variables. The gradual disappearance of this second source of variance at scales larger than the correlation length is precisely what determines the crossover away the critical phase.

The higher cumulants are important indications of this crossover since they measure departures from a Gaussian distribution, so we give a few more recur- 
sions implied by the SRG branching process. The skewness, $c_{N, 3}(p)$, satisfies,

$$
c_{m n, 3}(p)=\mu_{n}\left(p_{m}\right) c_{m, 3}(p)+3 \sigma_{n}\left(p_{m}\right)^{2} \mu_{m}(p) \sigma_{m}(p)^{2}+c_{n, 3}\left(p_{m}\right) \mu_{m}(p)^{3},
$$

and the kurtosis, $c_{N, 4}(p)$, satisfies,

$$
\begin{aligned}
c_{m n, 4}(p)= & \mu_{n}\left(p_{m}\right) c_{m, 4}(p)+\sigma_{n}\left(p_{m}\right)^{2}\left(4 \mu_{m}(p) c_{m, 3}(p)+3 \sigma_{m}(p)^{4}\right) \\
& +3 c_{n, 3}\left(p_{m}\right) \mu_{m}(p)^{2} \sigma_{m}(p)^{2}+c_{n, 4}\left(p_{m}\right) \mu_{m}(p)^{4} .
\end{aligned}
$$

On the right side of each of these recursions, the first term represents the additivity of the cumulants of the mass within each cell over the expected number of occupied cells, as in sums of independent variables. The remaining terms represent additional contributions due to fluctuations in the number of occupied cells. As we elucidate below, the phase transition occurs as a result of the competition between these two kinds of fluctuations, representing shortrange and long-range correlations, respectively.

\subsection{Crossover of Probability Distributions}

As discussed in section 2, large systems near the critical point which have the same ratio, $L / \xi(p)$, are in the same stage of crossover, i.e. they are statistically identical. This situation can be conveniently arranged within the formalism of the SRG, where the analogous ratio is $L / \xi^{(n)}(p)$ : Different systems are in the same stage of crossover if they have the same cell occupation probability on the largest cells, i.e. at the highest level of coarse-graining. In particular, a sequence of systems of increasing size can be frozen in the same state of crossover by adjusting $p$ at each stage of recursion to set the occupation probability on the largest cells to a given constant, $0<\eta<1$, which acts as the finite-size scaling variable.

For example, if the cells have reached size $m=n^{j}$ for a system of size $N=$ $n^{j+1}$, then we adjust $p$ to the following value

$$
p=R_{n}^{-j}(\eta)=R_{n}^{-1} \circ R_{N}^{-1} \circ \ldots R_{N}^{-1}(\eta)
$$

(the inverse of $R_{n}(p)$ applied $j$ times). This choice ensures that the correlation length for the system is $\xi^{(n)}(\eta)$ in units of the largest cell length. In other words, the finite-size scaling variable is held constant,

$$
\frac{\xi^{(n)}(p)}{L}=\frac{\xi^{(n)}(\eta) n^{j / d}}{L}=\frac{\xi^{(n)}(\eta)}{b},
$$


where $n=b^{d}$ and $N=L^{d}$.

The crossover from subcritical to critical to supercritical is controlled by varying $\eta$ from 0 to $p_{c}^{(n)}$ to 1 , respectively. For a given value of $\eta$, if we define,

$$
\hat{h}_{n^{j+1}}(\eta, s)=\hat{f}_{n^{j}}\left(R_{n}^{-j}(\eta), s\right)
$$

then the SRG takes the form,

$$
\begin{aligned}
\hat{h}_{n}(\eta) & =\hat{f}_{n}(\eta) \\
\hat{h}_{n^{2}}(\eta) & =\hat{f}_{n}(\eta) \circ \hat{f}_{n}\left(R_{n}^{-1}(\eta)\right) \\
\hat{h}_{n^{3}}(\eta) & =\hat{f}_{n}(\eta) \circ \hat{f}_{n}\left(R_{n}^{-1}(\eta)\right) \circ \hat{f}_{n}\left(R_{n}^{-2}(\eta)\right) \\
& \vdots \\
\hat{h}_{n^{j+1}}(\eta) & =\hat{f}_{n}(\eta) \circ \hat{f}_{n}\left(R_{n}^{-1}(\eta)\right) \circ \ldots \circ \hat{f}_{n}\left(R_{n}^{-j}(\eta)\right)
\end{aligned}
$$

in the cumulant-generating-function representation. By construction, the inverse iteration of the occupation probability converges to the critical fixed point,

$$
\lim _{j \rightarrow \infty} R_{n}^{-j}(\eta)=p_{c}^{(n)}
$$

for $0<\eta<1$, so the iteration for $\hat{h}_{N}(\eta, s)$ approaches a stationary functional iteration, which has a well defined limit (after an appropriate rescaling, discussed below). If we next take the large-cell limit, $n \rightarrow \infty$, then convergence is attained because the first term in the resulting infinite product tends to the true distribution inside a large cell of size $n \rightarrow \infty$, while each of the remaining terms tends toward a constant, $\hat{h}_{n}\left(p_{c}^{(n)}, s\right)$, because (again, by construction) $\lim _{n \rightarrow \infty} R_{n}^{-j}(\eta)=p_{c}^{(n)}$. Therefore, in the large-cell limit the $S R G$ produces asymptotic approximations of scaling functions and probability distributions in the crossover regime.

\subsection{Finite-Size Scaling Functions}

An analytical formula for the scaling function of the $k$ th cumulant,

$$
c_{L^{d}, k}(p) \sim\left(L^{D_{f}^{(n)}}\right)^{k} \Phi_{k}^{(n)}\left(\frac{L}{\xi^{(n)}(p)}\right)
$$


can be derived from the $n$-cell SRG. The trick is to choose $\eta$ according to Eq. (35) and thus cast the scaling function in the equivalent form,

$$
\Psi_{k}^{(n)}(\eta)=\Phi_{k}^{(n)}\left(\frac{b}{\xi^{(n)}(\eta)}\right)
$$

Although the subcritical $\left(p<p_{c}^{(n)}\right)$ and supercritical $\left(p>p_{c}^{(n)}\right)$ scaling functions are different in terms of the variable $x=L / \xi^{(n)}(p)$, there is a single, continuous scaling function in terms of the variable $\eta \in[0,1]$. From the auxiliary function, $\Psi_{k}^{(n)}(\eta)$, the usual scaling function for the $k$ th moment, $\Phi_{k}^{(n)}(x)$, can be determined from Eq. (40), once the appropriate $n$-cell correlation length, $\xi^{(n)}(p)$, is measured (e.g. as in Fig. 1).

The auxiliary scaling function, $\Psi_{k}^{(n)}(\eta)$, is easily calculated (at least numerically) from Eq. (37). For example, consider the case of the mean, $k=1$. After $j$ iterations of the SRG, an approximation of the scaling function, $\Psi_{1}^{(n)}(\eta)$, is given by

$$
\frac{\mu_{n^{j+1}}(p)}{\mu_{n}\left(p_{c}^{(n)}\right)^{j+1}}=\frac{\mu_{n}(\eta)}{\mu_{n}\left(p_{c}^{(n)}\right)} \cdot \frac{\mu_{n}\left(R_{n}^{-1}(\eta)\right)}{\mu_{n}\left(p_{c}^{(n)}\right)} \cdot \frac{\mu_{n}\left(R_{n}^{-2}(\eta)\right)}{\mu_{n}\left(p_{c}^{(n)}\right)} \cdot \ldots \cdot \frac{\mu_{n}\left(R_{n}^{-j}(\eta)\right)}{\mu_{n}\left(p_{c}^{(n)}\right)}
$$

where the rescaling factors in the denominator are chosen so that

$$
\Psi_{1}^{(n)}\left(p_{c}^{(n)}\right)=\Phi_{1}^{(n)}(0)=1
$$

This is also required for convergence as $j \rightarrow \infty$ (with $\eta$ fixed) since $R_{n}^{-j}(\eta) \rightarrow$ $p_{c}^{(n)}$. In this limit, we obtain a formula for the $n$-cell SRG approximation of the infinite-system scaling function,

$$
\Psi_{1}^{(n)}(\eta)=\prod_{j=0}^{\infty} \frac{\mu_{n}\left(R_{n}^{-j}(\eta)\right)}{\mu_{n}\left(p_{c}^{(n)}\right)}
$$

It is straightforward to prove that this infinite product converges if $\mu_{n}^{\prime}\left(p_{c}^{(n)}\right)>$ 0 and $R_{n}^{\prime}\left(p_{c}^{(n)}\right)>0$, as required for the critical exponents to be finite and nonzero.

By comparing Eq. (39) and Eq. (41), we have

$$
L^{D_{f}^{(n)}}=n^{D_{f}^{(n)} j / d}=\mu_{n}\left(p_{c}^{(n)}\right)^{j}
$$

which yields a simple estimate of the fractal dimension,

$$
D_{f}^{(n)}=d \log _{n} \mu_{n}\left(p_{c}^{(n)}\right) .
$$


It is apparent that this estimate of the fractal dimension converges to the correct value in the large-cell limit, as long as $p_{c}^{(n)} \rightarrow p_{c}$, because it is equivalent to the usual finite-system estimate used in numerical simulations, $\mu_{n}\left(p_{c}\right) \sim$ $n^{D_{f} / d}$. Equation (45) has also been derived by Hovi and Aharony using similar methods, albeit valid only at the critical point [13] (see also below). Here, however, we also predict scaling functions and probability distributions for the complete crossover between phases.

As $n \rightarrow \infty$, the first term in the infinite product of Eq. (43) converges to the true scaling function, and all other terms converge to unity, since $\lim _{n \rightarrow \infty} R_{n}^{-1}(\eta)=p_{c}^{(n)}$ for $0<\eta<1$ according to Eq. (5). This suggests that the SRG approximation of the scaling function converges in the large-cell limit. A very similar convergence proof is carried out in detail in Part II for an infinite-product formula for the strength of the infinite cluster.

\subsection{A Simple Example}

To illustrate the SRG approach, we compute estimates of finite-size scaling functions for the total mass of clusters spanning a square system in one direction, using the very simple four-cell scheme introduced above in section 2.4. In this case, the SRG is based on the conditional probability generating function,

$$
\bar{f}_{4}(p, z)=\frac{2 p^{2} q^{2} z^{2}+4 p^{3} q z^{3}+p^{4} z^{4}}{R_{4}(p)},
$$

where $R_{4}(p)$ is defined in Eq. (11), which yields the following polynomials for the moments,

$$
\mu_{4, k}(p)=\frac{2^{k+1} p^{2} q^{2}+3^{k} 4 p^{3} q z^{3}+4^{k} p^{4}}{R_{4}(p)} .
$$

From these expressions it is straightforward to compute (at least numerically) the auxiliary scaling functions $\Psi_{k}^{(n)}(\eta)$, but it would also be necessary to calculate the correlation length, $\xi^{(n)}(p)$ to obtain the usual scaling functions, $\Phi_{k}^{(n)}(x)$.

In practice, it is easier to iterate the cumulant recursions (numerically) starting from two particular values of $p$ very close to $p_{c}^{(n)}$ (one above and one below), as in Fig. 1, and then rescale by appropriate values of $\xi^{(n)}(p)$ for the chosen values of $p$. This method effectively samples the true continuous scaling functions at discrete points $b^{j} / \xi^{(n)}(p)$, which are very closely spaced (in log coordinates). For our four-cell example, the results of this method are shown in Fig. 3. 

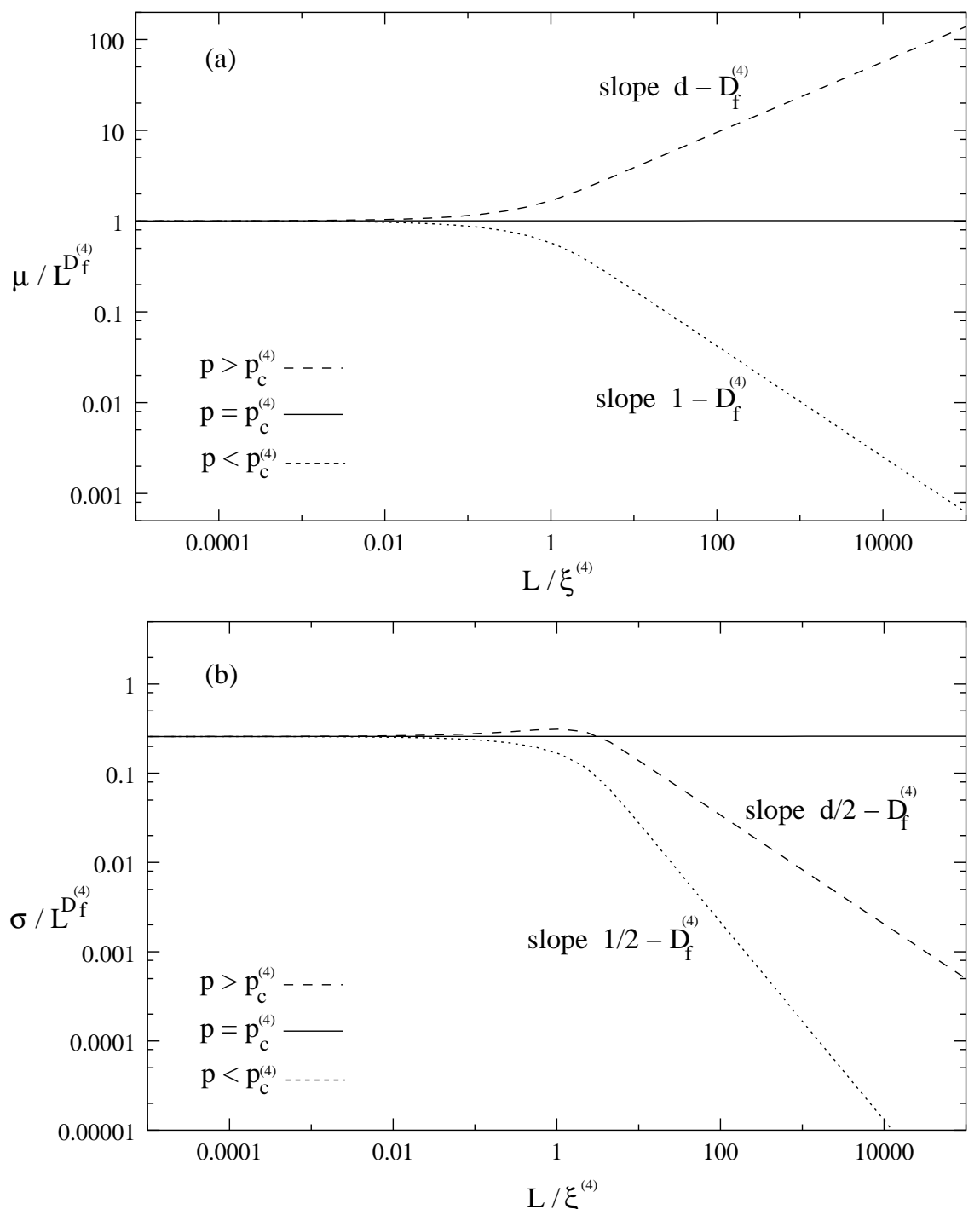

Fig. 3. Four-cell SRG estimates of crossover functions for (a) the mean and (b) the standard deviation of the total mass of clusters spanning a square system in one direction.

The scaling functions in Fig. 3 exhibit a smooth crossover from one power law to another (straight lines on a log-log plot) as the system size exceeds the correlation length, which is the correct qualitative behavior. The quantitative accuracy of these particular approximations is not very good, however, because even the fractal dimension (limiting slope) is poorly described. For this example, Equation $(45)$ predicts $D_{f}^{(n)} \approx 1.611$, for the mass of spanning clusters, compared to the presumably exact value, $D_{f}=91 / 48 \approx 1.896$.

As with the critical exponent, $\nu$, four cells $(b=2)$ is not enough to reasonably approximate the fractal dimension, $D_{f}$, but these estimates improve very quickly with increasing cell size. For example, Equation (45) predicts fractal dimensions for various structural quantities on the square bond lattice with less one percent error using remarkably small cells $(b \leq 4)$ [13]. Likewise, 
preliminary numerical results with the SRG using larger cells on the square site lattice also suggest that the approximation of scaling functions improves quickly with cell size, concomitant with the improvement in describing the fractal dimension. This ongoing work will be reported elsewhere, but at least the analysis here predicts convergence in the large-cell limit (provided that the scaling functions actually exist).

\section{$5 \quad$ The Supercritical Fixed Point}

\subsection{Asymptotic Independence}

A detailed analysis of the fixed points of the SRG will be presented in upcoming parts of this series, so here in the remaining sections we simply give the basic ideas.

Above the critical point of the RG, $p>p_{c}^{(n)}$, once the linear cell size exceeds the correlation length, $m^{1 / d} \gg \xi^{(n)}(p)$, the renormalized cell occupation probability approaches the supercritical fixed point, $p_{m} \rightarrow 1$. In this limit, the sums in Eq. (16) no longer have a random number of terms because $X_{n}(1)$ is equal to a constant, $x_{n}^{+}$, with probability one, i.e.

$$
\bar{f}_{n}(1, z)=z^{x_{n}^{+}}, \quad \tilde{f}_{n}(1, s)=e^{x_{n}^{+} s}, \text { and } \hat{f}_{n}(1, s)=x_{n}^{+} s
$$

The suppression of randomness in the number of "children" of the SRG branching process corresponds to the removal of hierarchical correlations at scales larger than the correlation length. In the infinite-system limit, independent contributions of mass from all cells are simply accumulated at each level of recursion. In other words, the SRG at the supercritical fixed point reduces to a non-random sum of independent random variables,

$$
X_{m n}(p) \sim \sum_{i=1}^{x_{n}^{+}} X_{m}^{(i)}(p)
$$

For example, at scales larger than the correlation length in the supercritical phase, all $x_{n}^{+}=n=b^{d}$ cells make independent contributions to the total mass of spanning clusters, while only a straight line of $x_{n}^{+}=b$ cells contributes to

the minimum chemical distance in a hypercubic system. (The former case is illustrated in the top row of Figure 2.) 


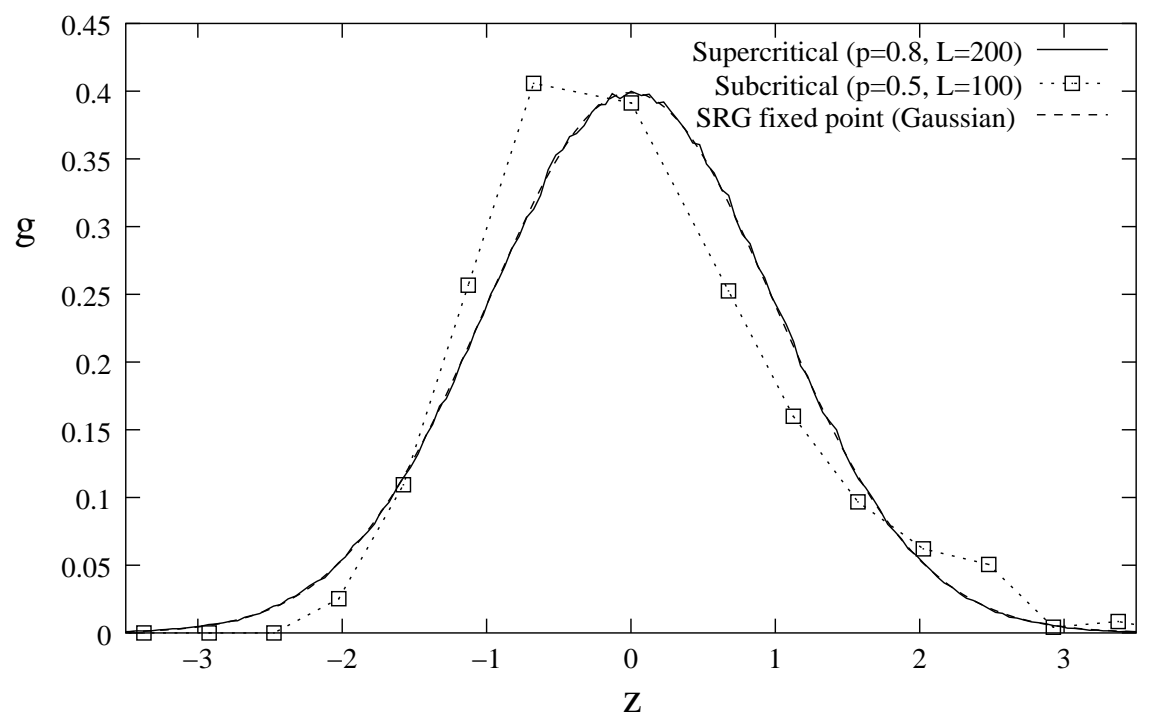

Fig. 4. Supercritical and subcritical probability distributions, $g(z)$, for the total mass of clusters spanning a square system in either direction (scaled to have zero mean and unit variance). Numerical simulation data for square-lattice site percolation is compared with the analytical prediction of the SRG at the off-critical fixed points, i.e. the Gaussian, $e^{-z^{2} / 2} / \sqrt{2 \pi}$. The numerical data reflects averaging over 10 million samples for a system of size $N=200 \times 200$ for the supercritical case and 20 million samples of size $N=100 \times 100$ for the subcritical case.

\subsection{Central Limit Theorem}

It is well known that non-random sums of independent random variables obey the Central Limit Theorem (CLT), which is characterized by a Gaussian limiting distribution with linear scaling of the mean and variance [32]. In the case of the SRG, "the CLT holds" means that the distribution of the scaled random variable

$$
Z_{N}=\frac{X_{N}(p)-\mu_{N}(p)}{\sigma_{N}(p)}
$$

converges to a standard normal distribution (i.e. a Gaussian with zero mean and unit variance). If equality holds in Eq. (49) and thus Eq. (48) applies, then the CLT is straightforward to prove from the cumulant generating function representation,

$$
\hat{f}_{m n}(s, p)=x_{+} \hat{f}_{m}(s, p)
$$

which implies the additivity of cumulants (the crucial step in most proofs of the CLT). This recursion is only asymptotically valid, however, so it is not clear a priori that the convergence is fast enough to ensure that the CLT holds. 
Nevertheless, in Part II it is shown that the CLT does in fact hold for the supercritical fixed point of the SRG. This result is consistent with Newman's proofs of the CLT for the mass of finite fragments of the infinite cluster [26,65,28]. The SRG prediction is also in excellent agreement with our own new simulation results (using the methods of Ref. [20]) for the total mass of clusters spanning a large square system in either direction, shown in Fig. 4.

\section{The Subcritical Fixed Point}

For $p<p_{c}^{(n)}$, the occupation probability renormalizes to zero because the spanning probability tends to zero (exponentially) once the cell size exceeds the correlation length. In this limit, all cells become "unoccupied", and hence spanning clusters are extremely rare. When spanning clusters do occur, however, they tend to include the minimum number of cells, $x_{n}^{-}$, in a straight line across the system $\left(x_{n}^{-}=b\right)$, as illustrated in Fig. 2. Indeed, this intuition is supported by the SRG for the conditional distribution of spanning cluster masses: At the subcritical fixed point, the SRG again reduces to a non-random sum of random variables,

$$
X_{m n}(p) \sim \sum_{i=1}^{x_{n}^{-}} X_{m}^{(i)}(p)
$$

since $X_{n}(0)=x_{n}^{-}$with probability one.

As in the supercritical case, this suggests that the CLT holds. The simulation data in Fig. 4 is also consistent with this claim, although it is difficult to generate enough subcritical spanning clusters to attain good convergence of the distribution. (Much better convergence could presumably be achieved using a "go-with-the-winners" algorithm [66].) To the author's knowledge, the validity of the CLT for various quantities conditional on spanning in subcritical percolation has not previously been reported.

Since spanning clusters are very rare in the subcritical regime, a much better order parameter for the entire phase transition in finite systems is the mass of the largest cluster [35-37,19,20]. At the subcritical fixed point, the mass distribution of the largest cluster does not obey the CLT, and instead the Fisher-Tippett limit theorem of extreme statistics holds $[19,20]$. On the other hand, the CLT does hold for the largest-cluster mass at the supercritical fixed point because the largest cluster and various spanning clusters are all the same in the supercritical limit. To unify the present theory with these intriguing results revealing subcritical/supercritical asymmetry, in Part IV the SRG is extended to the more subtle case of the mass of the largest cluster (which may 
not span).

\section{$7 \quad$ The Critical Fixed Point}

\subsection{Complete Statistical Self-Similarity}

At the critical fixed point, $p=p_{c}^{(n)}$, the SRG reduces exactly to

$$
X_{m n}=\sum_{i=1}^{X_{n}} X_{m}^{(i)},
$$

which asserts that, at every scale, the random cluster mass $X_{m n}$ in a system of $N=m n$ sites has the same statistics as the sum of independent mass contributions $X_{m}^{(i)}$ from a random number $X_{n}$ out of $n$ cells of size $m$. (In this section, we will drop the $p$ arguments in our notation with the understanding that $p=p_{c}^{(n)}$.) As was recently first pointed out by Hovi and Aharony [13], the $n$-cell iteration of Eq. (53), with the distribution of $X_{n}$ fixed in the upper limit of the random sums, corresponds to a stationary Watson-Galton branching process $[67,33,34,32]$. This classical discrete branching process describes the growth of a population of individuals, each of whom has a random number of children, $X_{n}$, sampled independently from the same distribution.

The self-similar iteration of Eq. (53),

$$
X_{m^{2}}=\sum_{i=1}^{X_{m}} X_{m}^{(i)},
$$

starting from the initial condition $X_{n}$ (where $N=n^{2^{j}}$ ) corresponds to a hierarchical sampling of the Watson-Galton process: The distribution of grandchildren is that of the children's children, the distribution of great, great grandchildren is that of the grandchildren's grandchildren, etc. In this form, the SRG at the critical fixed point clearly expresses statistical self-similarity at all length scales because the coarse-grained mass "on the cells" (the random number of terms in the sum) has the same distribution as the random mass contributions from each cell.

In terms of cumulant generating functions, the SRG at the critical fixed point is simply a functional iteration,

$$
\hat{f}_{m n}=\hat{f}_{n} \circ \hat{f}_{m}
$$


starting from the initial condition $\hat{f}_{n}(s)$. From Eq. (30), this implies that the mean scales with system size, $N=L^{d}=n^{j}$, as a power law,

$$
\mu_{n^{j}}=\left(\mu_{n}\right)^{j}=L^{D_{f}^{(n)}}=\left(n^{D_{f}^{(n)} / d}\right)^{j}
$$

where the $n$-cell estimate of the fractal dimension is given once again by Eq. (45).

(From Eq. (56), we see that $\mu_{n}>1$ is a necessary condition for the expected value of the critical order parameter to grow with system size, or equivalently, for the fractal dimension to be positive. In the context of population growth, this is the case where the expected population size grows exponentially with the number of generations, e.g. a supercritical nuclear reaction. Conversely, the case, $\mu_{n}<1$, models exponentially fast extinction of a population, e.g. a subcritical nuclear reaction. As such, probability theorists refer to these two types of branching processes as "supercritical" and "subcritical", respectively, and while the marginal case $\mu_{n}=1$ is naturally termed "critical" [32-34]. Unfortunately, this standard mathematical terminology is poorly suited here because the SRG at the critical fixed point is actually a "supercritical branching process". In fact, the non-stationary SRG branching process is actually "supercritical" for any thermodynamic phase since the expected value of the order-parameter always increases with system size.)

\subsection{The Fractal Central Limit Theorem}

Although the SRG corresponds to a new kind of non-stationary branching process, various existing mathematical results for simple Watson-Galton branching processes $[67,33,34]$ have direct relevance for the critical fixed point [13]. For example, the "central region" of the limiting distribution is at the same scale as the mean. In other words, the scaled random variable, $Z_{N}=X_{N} / \mu_{N}$, has a stationary limiting distribution,

$$
\lim _{j \rightarrow \infty} \operatorname{Prob} .\left(X_{n^{j}}<z \mu_{n^{j}}\right)=G^{(n)}(z)
$$

for some nontrivial function $G^{(n)}(z)$ (the $n$th SRG estimate of the true limiting distribution). This result, which can be derived from Eq. (55), explains why random fractals, such as critical spanning clusters in percolation, have very large mass fluctuations, comparable to the mean, in contrast to normal random objects, such as supercritical spanning clusters, which have "square-root fluctuations" (at the scale of the square root of the mean) as a consequence of the CLT. 


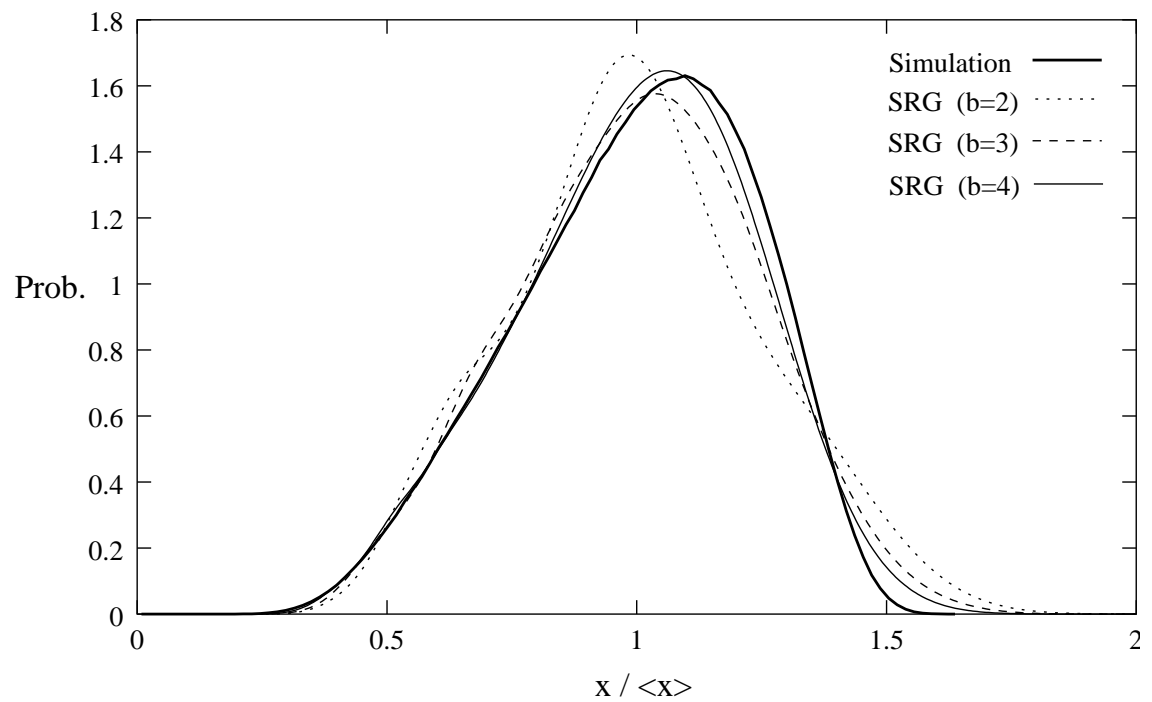

Fig. 5. Limiting probability distribution (scaled to have unit mean) for the total mass of clusters spanning a square system in one direction (solid line, obtained from simulations on a $300 \times 300$ site lattice with $p=0.592746 \approx p_{c}$ ) compared with $2 \times 2$, $3 \times 3$, and $4 \times 4$ SRG approximations (dashed lines, obtained by numerically iterating the exact recursions given in the main text).

In Part III, it is shown that for percolation quantities, the SRG critical fixed point, $G(z)=\lim _{n \rightarrow \infty} G^{(n)}(z)$, is generally not Gaussian. Its shape depends sensitively on macroscopic constraints, such as spanning or boundary conditions, although it is presumably universal for different lattices in the same embedding dimension. The fact that shape of the critical order-parameter distribution depends on macroscopic constraints, for arbitrarily large systems, is a striking signature of long-range correlations, which has apparently not been previously noted in the literature. This is also the first conclusion of the FCLT: In the central region, the limiting mass distribution of a random unifractal is non-Gaussian and depends sensitively on macroscopic constraints.

Preliminary numerical evidence indicates that satisfactory approximations of critical distribution functions can be obtained using remarkably small cells. These approximations are produced by numerically iterating the recursions given above, either for the probability density directly (which involves discrete convolutions) or for one of the generating functions (which involves simple multiplications followed by an inverse transform). In Fig. 5, we see that the very simple $n=2 \times 2$ cell scheme discussed above is surprisingly close to the results of large-scale simulations for clusters spanning a square site lattice in one direction. Even more striking is the good accuracy achieved using only slightly larger cells, $n=3 \times 3$ and $n=4 \times 4$. These approximations and others are analyzed in more detail in Part III. 
The SRG also predicts universal stretched-exponential decay for the tails of the critical limiting distributions,

$$
\log g(z) \sim \begin{cases}-A(z) z^{\hat{\delta}} & \text { as } z \rightarrow \infty \\ -B(z) z^{\hat{\eta}} & \text { as } z \rightarrow 0\end{cases}
$$

where $g(z)=G^{\prime}(z)$ is the limiting probability density function in the limit of infinite cell size, and $A(z)$ and $B(z)$ are periodic functions of $\log z$, which are essentially constant in practice [13]. (Note that stretched-exponential decay of the left tail as $z \rightarrow 0$ holds only if $x_{n}^{-}>1$.) The tail exponents, $\hat{\delta}$ and $\hat{\eta}$, can be expressed in terms of other critical exponents and do not depend on macroscopic constraints. If the mean scales like $\mu_{n}\left(p_{c}\right) \sim n^{\delta}$ and the largest possible value scales like $x_{n}^{+} \sim n^{\delta^{+}}$, then the exponent for the right tail is given by,

$$
\hat{\delta}=\frac{\delta^{+}}{\delta^{+}-\delta}
$$

Similarly, if the smallest possible value scales like $x_{n}^{-} \sim n^{\delta^{-}}$, then the exponent for the left tail is given by,

$$
\hat{\eta}=\frac{\delta^{-}}{\delta^{-}-\delta}
$$

In the case of spanning clusters in $d$ dimensions, we have $\delta=D_{f} / d, \delta^{+}=1$, and $\delta^{-}=1 / d$, in which case the tail exponents are,

$$
\hat{\delta}=\frac{d}{d-D_{f}}
$$

and

$$
\hat{\eta}=\frac{1}{1-D_{f}}
$$

These conclusions follow directly from classical mathematical theorems on Watson-Galton branching processes [33,34,67], as first pointed out by Hovi and Aharony [13]. Note that the co-dimension, $d-D_{f}$, governs the right tail, because rare, very large clusters have the dimension of the system $d$, rather than the normal fractal dimension, $D_{f}$. Similarly, the co-dimension, $D_{f}-1$, governs in the left tail, because rare, very small clusters (conditional on spanning) are roughly linear objects with dimension one. 


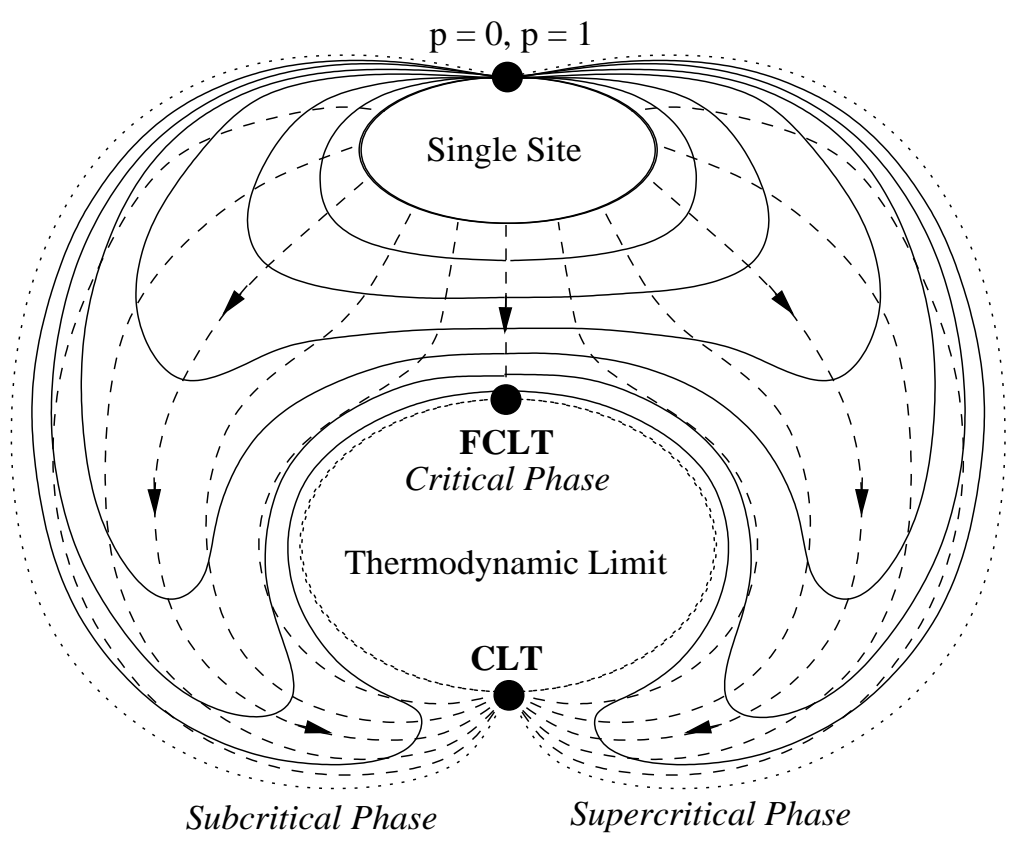

Fig. 6. Topology of the SRG flow for in the space of scaled probability distributions of any structural quantity conditional on spanning, such as the total cluster mass or the minimum chemical distance connecting two opposite boundaries.

These results lead us to the second general conclusion of the FCLT: Outside the central region, the limiting distributions of random unifractals have stretched exponential tails with universal exponents given by Eqs. (59)-(62). In ongoing work [31], the generality of the FCLT is explored by comparisons with percolation, random graphs, and the Ising model, and the subtle question of what happens above the upper critical dimension is also addressed.

\section{Discussion}

Combining the results of this article, we arrive at a fruitful intuitive picture of the SRG "flow" (dashed lines) in the space of probability distributions, as sketched in Fig. 6. As the system size is increased, the physical manifold $0 \leq p \leq 1$ (solid lines) is advected from the single-site manifold for $N=1$ toward the thermodynamic manifold for $N=\infty$ (interior dotted line). The SRG accurately describes the universal flow near the two thermodynamic fixed points and the crossover manifold connecting them, far away from the $p=0,1$ fixed point representing discreteness and non-universality. For a fixed value of $p \neq 0, p_{c}, 1$, at small sizes the system trajectory passes near the (unstable) critical fixed point where the FCLT holds and then crosses over toward the (stable) off-critical fixed point, where the CLT holds, once the system size exceeds the correlation length.

The significance of the SRG is both practical and fundamental. In real ap- 
plications, critical phenomena such as percolation occur in a finite systems, which are often small enough compared to the correlation length that finitesize effects are important. In such cases, the SRG provides a simple analytical or numerical method to approximate the mean, variance, and distribution of an order parameter for arbitrary system sizes and values of the control parameter, even when the correlation length and system size are comparable. These approximations should be fairly accurate as long as the correlation length and the system size are much larger than one.

Preliminary numerical results for percolation suggest that reasonable accuracy can be achieved with remarkably small cell sizes $(b>2)$. Developing the best small-cell approximations for various quantities in percolation and other statistical models will require more extensive comparisons with simulation data (as in upcoming parts of this series). Whenever reasonable small-cell schemes can be identified, however, they allow trivial calculations which may suffice to replace direct simulations in many applications. From a practical point of view, simple approximations for a broad range of conditions can be much more useful than exact results for certain limiting cases because models like percolation are always themselves only crude approximations of real physical systems.

The SRG also provides basic insights into critical phenomena and random fractals. The connection with branching processes gives a simple "populationgrowth" picture of how crossover occurs in a continuous phase transition. The fixed points of the SRG also explain the nature of fluctuations in different thermodynamic phases, e.g. governed by the CLT and the FCLT. These conclusions have been reached here in the specific context of percolation, which is perhaps the simplest case, but it should be possible to extend the SRG to other critical phenomena, such as the Ising model, by postulating and analyzing appropriate non-stationary branching processes expressing statistical self-similarity.

\section{Acknowledgments}

The author gratefully acknowledges L. K. Bazant, J.-P. Bouchaud, L. P. Kadanoff, P. Sen, and H. E. Stanley for useful discussions, and D. Harmon for also providing the small-cell SRG results presented in some of the figures. 


\section{References}

[1] H. E. Stanley, Introduction to Phase Transitions and Critical Phenomena (Oxford University Press, 1971).

[2] S.-K. Ma, Modern Theory of Critical Phenomena (Perseus Publishing, Cambridge, MA, 1976).

[3] N. Goldenfeld, Lectures on Phase Transitions and the Renormalization Group (Perseus Books, Reading, MA, 1992).

[4] L. P. Kadanoff, Statics, Dyanmics and Renormalization (World Scientific, Singapore, 2000).

[5] A. Bunde and S. Havlin, in Fractals and Disordered Systems, edited by A. Bunde and S. Havlin (Springer, 1996), pp. 58-113, 2nd ed.

[6] B. Hughes, Random Walks and Random Environments, vol. II (Clarendon Press, Oxford, 1995).

[7] D. Stauffer and A. Aharony, Introduction to Percolation Theory (Taylor and Francis, London, 1994), 2nd ed.

[8] S. Havlin, B. Trus, G. H. Weiss, and D. Ben-Avraham, J. Phys. A. 18 (1985) L247.

[9] S. Havlin and D. Ben-Avraham, Adv. Phys. 36, 695 (1987).

[10] S. Havlin, J. E. Keifer, F. Leyvraz, and G. H. Weiss, J. Stat. Phys. 47, 173 (1987).

[11] A. U. Neumann and S. Havlin, J. Stat. Phys. 52, 203 (1988).

[12] J.-P. Hovi and A. Aharony, Fractals 3, 4553 (1995).

[13] J.-P. Hovi and A. Aharony, Phys. Rev. E 56, 172 (1997).

[14] N. V. Dokholyan, Y. Lee, S. Buldyrev, S. Havlin, P. R. King, and H. E. Stanley, J. Stat. Phys. 93, 603 (1998).

[15] N. V. Dokholyan, S. Buldyrev, S. Havlin, P. R. King, Y. Lee, and H. E. Stanley, Physica A 266, 55 (1999).

[16] J. S. Andrade, S. V. Buldreyev, N. V. Dokholyan, S. Havlin, P. R. King, Y. Lee, G. Paul, and H. E. Stanley, Phys. Rev. E 62, 8270 (2000).

[17] G. Paul, S. Havlin, and H. E. Stanley, cond-mat/0203092.

[18] P. Sen, Int. J. Mod. Phys. C 10, 747 (1999).

[19] P. M. Duxbury and P. L. Leath, J. Phys. A 20, L411 (1987).

[20] M. Z. Bazant, Phys. Rev. E 62, 1660 (2000). 
[21] M. I. Zeifman and D. Ingman, J. Appl. Phys. 88, 76 (2000).

[22] P. Sen, J. Phys. A, 34, 8477 (2001).

[23] P. R. King, J. S. Andrade, S. Buldyrev, N. V. Dokholyan, S. Havlin, Y. Lee, and H. E. Stanley, Physica A 266 (1999).

[24] Y. Lee, J. S. Andrade, S. Buldyrev, N. V. Dokholyan, S. Havlin, P. R. King, G. Paul, and H. E. Stanley, Phys. Rev. Lett. (1999).

[25] S. Torquato, Random Heterogeneous Materials (Springer, New York, 2000).

[26] C. M. Newman, Commun. Math. Phys. 74, 119 (1980).

[27] C. M. Newman and L. S. Schulman, J. Stat. Phys. 26, 613 (1981).

[28] C. M. Newman, Commun. Math. Phys. 91, 75 (1983).

[29] J. T. Cox and G. Grimmett, J. Stat. Phys. 25, 237 (1981).

[30] J. T. Cox and G. Grimmett, Ann. Probab. 12, 514 (1984).

[31] M. Z. Bazant and J.-P. Bouchaud, in preparation.

[32] W. Feller, An Introduction to Probability Theory and Its Applications, vol. II (Wiley, New York, 1971), 2nd ed.

[33] T. E. Harris, The Theory of Branching Processes (Springer-Verlag, Berlin, 1963).

[34] K. B. Athreya and P. E. Ney, Branching Processes (Springer-Verlag, New York, 1972).

[35] D. Stauffer, Z. Phys. B 32, 89 (1980).

[36] F. Family and A. Coniglio, J. Phys. A 13, L403 (1980).

[37] A. Margolina and H. J. Herrmann, Physics Letters 104A, 295 (1984).

[38] G. I. Barenblatt, Dimensional Analysis (Gordon and Breach, New York, 1987).

[39] P. J. Reynolds, W. Klein, and H. E. Stanley, J. Phys. C 10, L167 (1977).

[40] P. J. Reynolds, H. E. Stanley, and W. Klein, J. Phys. A 11, L199 (1978).

[41] P. J. Reynolds, H. E. Stanley, and W. Klein, Phys. Rev. B 21, 1223 (1980).

[42] A. P. Young and R. B. Stinchcombe, J. Phys. C 8, L535 (1975).

[43] S. Kirkpatrick, Phys. Rev. B 15, 1533 (1977).

[44] Y. Yuge and C. Murase, J. Phys. A 11, L83 (1978).

[45] Y. Yuge, Phys. Rev. B 18, 1514 (1978).

[46] M. E. J. Newman and R. M. Ziff, Phys. Rev. Lett. 85, 4104 (2000). 
[47] J.-P. Hovi and A. Aharony, Phys. Rev. E 53, 235 (1996).

[48] R. P. Langlands, C. Pichet, P. Pouliot, and Y. Saint-Aubin, J. Stat. Phys. 67, 553 (1992).

[49] P. Grassberger, J. Phys. A 25, 5475 (1992).

[50] R. M. Ziff, Phys. Rev. Lett. 669, 2670 (1992).

[51] R. P. Langlands, P. Pouliot, and Y. Saint-Aubin, Ann. Math. Stat. 30, 1 (1994).

[52] D. Stauffer, J. Adler, and A. Aharony, J. Phys. A 27, L475 (1994).

[53] U. Gropengiesser and D. Stauffer, Physica A 210, 320 (1994).

[54] C. K. Hu, J. Phys. A 27, L813 (1994).

[55] C.-K. Hu and J.-A. Chen, J. Phys. A 28, L73 (1995).

[56] A. Aharony and J.-P. Hovi, Phys. Rev. Lett. 72, 1941 (1994).

[57] C. D. Lorenz and R. M. Ziff, J. Phys. A 31, 8147 (1998).

[58] R. M. Ziff, C. D. Lorenz, and P. Kleban, Physica A 266, 17 (1999).

[59] R. M. Ziff and M. E. J. Newman, preprint, cond-mat/0203496.

[60] J. L. Cardy, J. Phys. A 25, L201 (1992).

[61] D. P. Landau and K. Binder, A Guide to Monte Carlo Simulations in Statistical Physics (Cambridge University Press, 2000).

[62] H. W. Watson and F. Galton, J. Anthropol. Inst. Great Britain Ireland 4, 138 (1874).

[63] R. A. Fisher, The Genetical Theory of Natural Selection (Clarendon Press, Oxford, 1930).

[64] E. Schroedinger, Probability Problems in Nuclear Chemistry, vol. 51A (Proc. Roy. Irish Acad., 1945).

[65] C. M. Newman and A. L. Wright, Ann. Probab. 9, 671 (1981).

[66] P. Grassberger and W. Nadler (2000), cond-mat/0010265.

[67] T. E. Harris, Ann. Math. Stat. 41, 474 (1948). 